\title{
RISK OF FATAL REAR-END COLLISIONS: IS THERE MORE TO IT THAN ATTENTION?
}

\author{
John M. Sullivan and Michael J. Flannagan \\ Human Factors Division \\ University of Michigan Transportation Research Institute \\ 2901 Baxter Road \\ Ann Arbor, Michigan,USA \\ E-mail: jsully@umich.edu
}

\begin{abstract}
Summary: Rear-end collisions predominantly occur in the daytime under clear, unobstructed viewing conditions and usually involve a lead vehicle that is stopped at the time of collision. These facts suggest that driver inattention plays a significant causal role in rear-end collisions, and mitigation efforts have therefore focused largely on development of warning technologies to alert drivers of an impending crash. However, we note that this pattern of crash data should not lead to the conclusion that drivers have special difficulty avoiding rear-end collisions in broad daylight. Nor should it be concluded that other "environmental" factors do not influence driving behavior to increase rear-end crash risk. Crash frequency is determined both by the inherent risk in the driving task and by the frequency of driver exposure to conditions in which a crash is possible. When exposure level is equated across conditions which differ in ambient light level, we find that rear-end collisions appear to be more than twice as likely in darkness than in daylight.
\end{abstract}

\section{INTRODUCTION}

According to the NHTSA's General Estimates System, in 2001 there were an estimated 1.9 million police-reported rear-end crashes in the United States, representing about $30 \%$ of a total of 6.3 million crashes. In contrast, rear-end crashes comprised only about $5 \%$ of all fatal crashes in 2001, suggesting that while numerous, rear-end crashes are not especially lethal. Nonetheless, rear-end crashes represent a considerable national cost and there is keen interest in developing effective countermeasures for this type of crash.

A detailed breakdown of the causal analysis of crash data taken from the Indiana Tri-Level Study (Treat et al., 1979) suggested that, especially for circumstances in which the lead vehicle is stopped (LVS), the majority of rear-end collisions (approximately 68\%) are caused by driver inattention or distraction (Knipling, Wang, \& Yin, 1993). This analysis was further supported by a follow-up causal analysis of the 1991 National Accident Sampling System (NASS) Crashworthiness Data System (CDS) in which driver inattention was implicated in $66.3 \%$ (weighted) of the rear-end crashes (Knipling, Mironer et al., 1993). Notably, factors related to environmental conditions (icy roads, fog, rain, obscured vision) appeared to play only a small causal role. Less than $10 \%$ of the Tri-Level cases, and less than $4 \%$ of weighted cases taken from the analysis of the 1991 CDS data, were attributed to environmental conditions. The apparently strong causal relationship between driver inattention and rear-end collision also appeared to be further supported by the fact that $76.5 \%$ of rear-end collisions occurred in daylight and $90 \%$ occurred on straight roadways (Knipling, Wang et al., 1993). Thus, the striking drivers in rearend crashes appear to be profoundly unaware of their immediate peril despite the generally 
favorable environmental conditions. Mitigation efforts have thus focused largely on the development of warning technologies to alert drivers of impending crashes.

Care should be taken not to over-interpret these crash facts and draw conclusions from them about the role of human attention. For example, it should not be concluded that inattentiveness is more common during the daytime on straight roads. Instead, it must be recognized that there are more opportunities for rear-end collisions during the daytime and on straight roadways. Rear-end collisions occur between a minimum of two vehicles: often one following another down a roadway at relatively close proximity. This circumstance is obviously less likely to occur at 03:00 h than at 17:00 h. Thus traffic density surely contributes to the observed pattern of rearend crashes and should not be mistaken for drivers' attentional processes. While it is appropriate and practical to design countermeasures engineered to match the circumstances in which rearend collisions frequently occur, the frequency statistics alone do not establish what characteristics of the driving situation affect a driver's performance to increase the risk of a rearend collision.

This paper takes a closer look at the role of ambient light in fatal rear-end collisions by analyzing the distribution of these crashes across the annual Daylight Saving Time (DST) transitions. The technique inspected the change in the distribution of fatal crashes during periods just before and just after DST changeovers at times of day that transition from darkness to daylight and vice versa. In comparing crash data from a one-hour clock window across the DST transition, we assumed that levels of many factors known to play a role in fatal crashes remain relatively constant, leaving ambient light level as a quasi-independent variable. For example, in the Detroit area, the one-hour period between 19:30 $\mathrm{h}$ and 20:30 $\mathrm{h}$ is dark before the spring changeover to DST, and light afterwards. An important assumption in this analysis is that traffic conditions (and hence, crash opportunities) are the same in the weeks immediately before and after the changeover to and from DST, as traffic is principally governed by clock time rather than by the position of the sun in the sky. Observed differences in crash levels between these two periods are thus likely to be related to the difference in ambient light level, and may be used to quantify the effect of light in fatal crashes.

Past analyses of increased crash risk in the dark have focused primarily on fatal pedestrian crashes, which are about four times more likely in the dark than in daylight (Sullivan \& Flannagan, 2002a, 2002b). A small, but reliable, heightened risk was also observed in these analyses for fatal crashes between motor vehicles (1.3 times more likely in the dark). In the current analysis, we follow up this finding by examining changes in the patterns of collisions between motor vehicles with special focus on disaggregating the crashes by collision type and with particular interest in rear-end collisions. Although environmental factors have been causally linked to only small numbers of rear-end crashes, they may nevertheless play a role in elevating the risk of rear-end crashes as long as factors related to crash opportunity are similar.

\section{METHOD}

Crash data from the Fatality Analysis Reporting System (FARS) of the National Highway Traffic Safety Administration (NHTSA) were selected from the 15-year period from 1987 to 2001. Cases selected for analysis straddled the daylight saving time changeover periods in time windows that abruptly changed from dark to light (or light to dark) across the time change. To perform the DST analysis, the exact start and end of civil twilight was computed for the dates of the spring and fall adjustments for DST for each county in the continental United States, for each 
of the 15 years covered in this analysis. Counties in states that do not observe DST, as well as counties split across time zones, were excluded.

Using the county times for the start and end of civil twilight, crash-record clock times were recoded to indicate if the crash occurred in the one-hour interval just after the end of civil twilight, in the evening. As shown in Figure 1, in the spring, this interval is dark before DST and light (or twilight) after the DST changeover. In the fall, the interval is light before the DST changeover, and dark following it. For the present analysis, crash records were taken from five weeks before and five weeks after the evening transitions in both the spring and fall, and tagged as falling into either a light or a dark period. Morning data were excluded from this analysis because ambient light fluctuates over the five-week morning intervals before and after the changeovers.

\section{Effect of Daylight Saving Time Changeover}

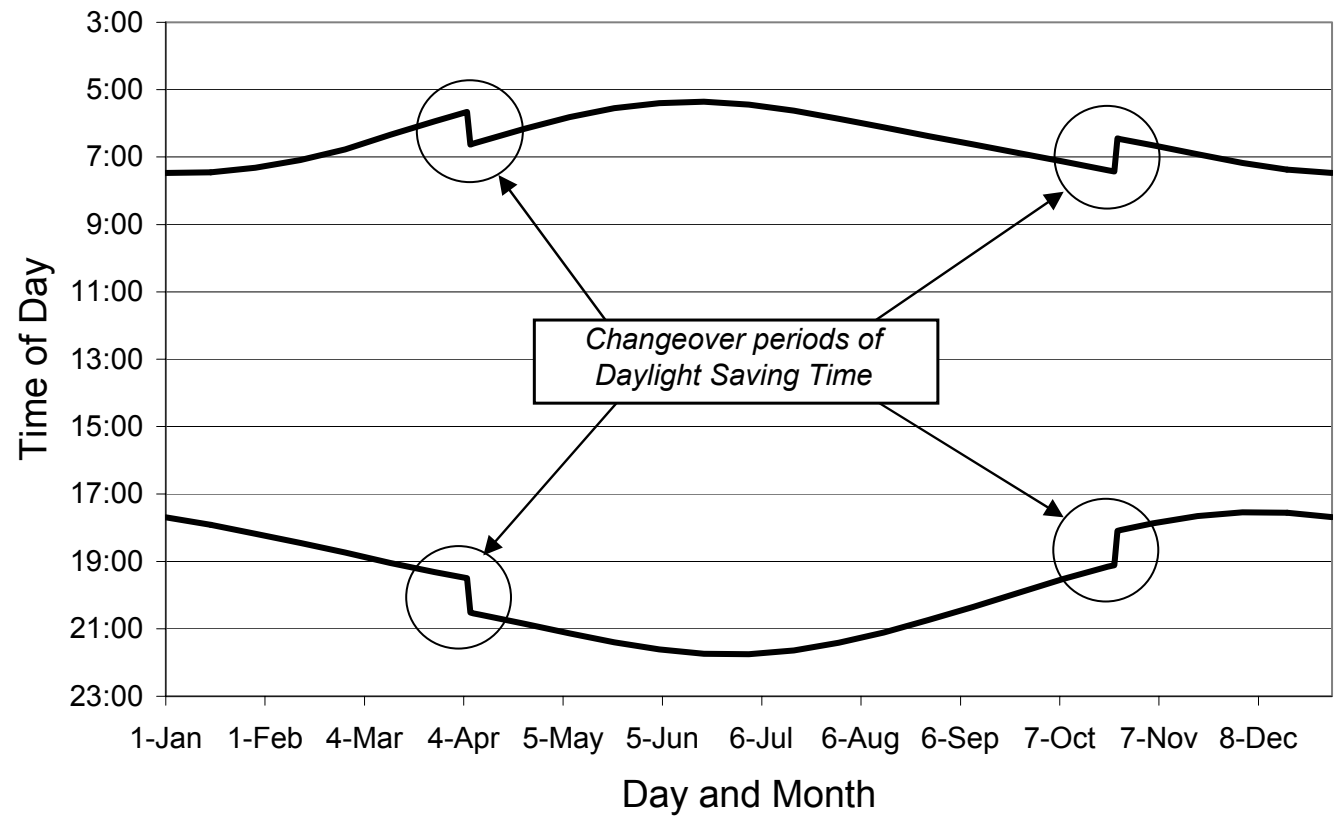

Figure 1. Daylight saving time changeover characteristics for spring and fall. The solid line is the clock time of the start (top) and end (bottom) of civil twilight throughout the year for the Ann Arbor/Detroit area (1997).

The magnitude of the risk was estimated by examining the ratio of crashes occurring during the dark period to those occurring during the light period. A simple null hypothesis would suggest that a crash is equally likely to occur in either the dark or light period, and should produce a dark-light ratio of one. A ratio greater than one indicates an increased risk in darkness; a ratio less than one indicates reduced risk in darkness.

In the first analysis, rear-end, front-end, and side-impact crashes involving two vehicles were compiled across the DST transitions. A further analysis was then conducted to examine the characteristics of rear-end collision risk as a function of vehicle role (striking, struck) and vehicle type (light vehicle, truck). 


\section{RESULTS}

Figure 2 illustrates the raw crash counts for each collision type across the one-hour evening time interval that changes from light to dark during the fall transition to standard time. The data are accumulated over 15 years (1987 to 2001) and show crash rates five weeks before and after the transition. Rear-end collisions change from an average rate of about 13 crashes in the light to an average of 37 in the dark, suggesting a dark-to-light crash ratio of about 3 to 1. For the remaining analyses, ratios are computed by pooling crash counts for spring and fall during the five-week dark and five-week light intervals before and after the transitions. Table 1 provides a breakdown of crash counts in dark and light for each crash type.

Overall, fatal rear-end collisions were less frequent than either side-impact collisions or frontend collisions, although they appeared to be more than twice as frequent in darkness as in daylight, suggesting that ambient light level may indeed play some role in affecting the likelihood of rear-end collisions.

\section{Fall PM Transition to Standard Time}

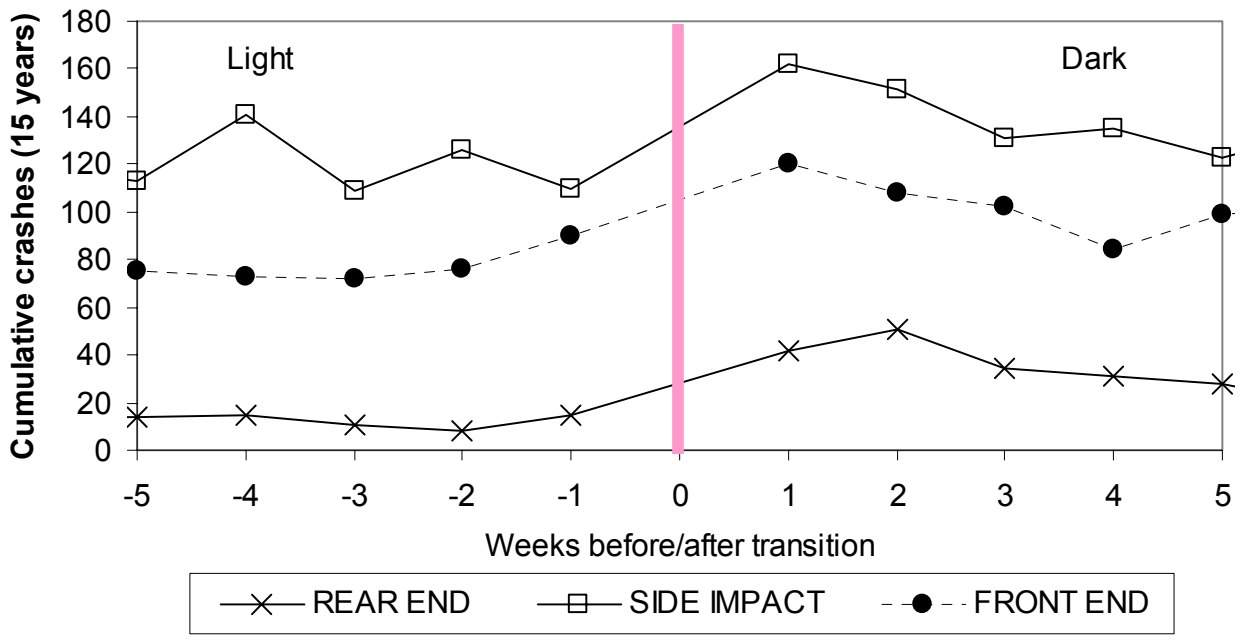

Figure 1. Crash counts in the weeks prior to and following the fall evening return to standard time. The right half of the figure shows crashes occurring during a one-hour time interval that is in daylight prior to the transition to standard time; the right half shows crashes in darkness.

Table 1. Accumulated crashes (and percent) in the dark and light intervals 5 weeks before and after the evening DST transition combined for both spring and fall

\begin{tabular}{lccc} 
& \multicolumn{2}{c}{ Ambient Light } & \multirow{2}{*}{$\begin{array}{c}\text { Dark/Light } \\
\text { Ratio }\end{array}$} \\
\cline { 2 - 3 } Crash Type & Dark & Light & 2.35 \\
\hline Rear-End & $289(70 \%)$ & $123(30 \%)$ & 1.09 \\
Side-Impact & $1099(52 \%)$ & $1008(48 \%)$ & 1.21 \\
Front-End & $785(55 \%)$ & $649(45 \%)$ & \\
\hline
\end{tabular}


In the next analysis, we examined the relationship between vehicle type (light vehicle or truck) and the role (striking or struck) played by each vehicle in the crash. Vehicle type categories were defined using the same scheme applied in previous rear-end collision analyses (Wiacek \& Najm, 1999): the category light vehicles included automobiles, light trucks, SUVs, and vans; the category trucks included medium and heavy trucks. Because of the small number of fatal bus and motorcycles crashes, they were not included in this analysis.

The breakdown of fatal crashes is shown in Figure 3. While fatal rear-end collisions involving striking light vehicles are three times more likely in the dark, there appears to be little difference in the striking-truck frequency during the dark and light periods. Especially noteworthy is that medium and heavy trucks appear to be over eight times as likely to be rear-ended in darkness as in daylight. This suggests that trucks are either more often unnoticed or somehow misperceived by drivers in darkness, leading to fatal collisions. That trucks are overrepresented as struck vehicles in nighttime crashes has been reported before (Green, Kubaki, Olson, \& Sivak, 1979), and this has prompted regulatory action in the last 10 years to make trucks more conspicuous, with some effect (Morgan, 2001).

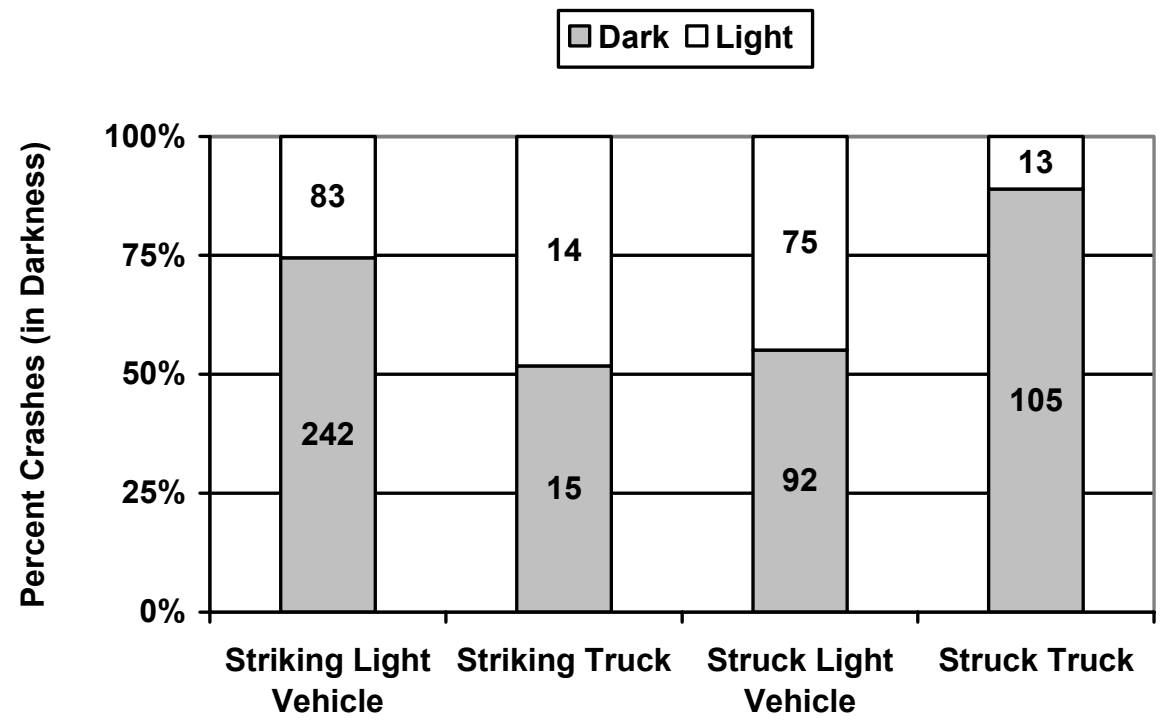

Figure 3. Fatal rear-end collisions during the spring and fall evening DST changeover period by vehicle type (light vehicle or truck) and role (striking or struck) in collision. The data were accumulated over 15 years for five-week periods before and after each changeover.

\section{CONCLUSION}

The relative risk of a fatal rear-end collision in darkness appears to be more than twice that in daylight. Although the added risk in darkness is substantial, it has perhaps been unnoticed until now because the volume of roadway encounters with stopped vehicles is far smaller in darkness than compared to that in daylight. We note that the discovery of such an environmental influence on fatal rear-end crash risk does not diminish the importance of attentional mechanisms in rearend collisions - in fact, allocation and reallocation of visual attention is likely influenced by target visibility. For example, detection of a looming object in the visual periphery is perhaps easier in daylight, permitting successful avoidance of a rear-end collision. Alternatively, perhaps with the reduced visibility of a forward vehicle, drivers are less compelled to keep their attention 
on the road. In any case, the results suggest that some degree of fatal rear-end crash mitigation might be possible if target visibility is enhanced.

\section{ACKNOWLEDGMENTS}

This research was supported by the University of Michigan Industry Affiliation Program for Human Factors in Transportation Safety. The current members of the Affiliation Program are: AGC America, Autoliv, Automotive Lighting, Avery Dennison, BMW, DaimlerChrysler, DBM Reflex, Denso, Exatec, Federal-Mogul, Fiat, Ford, GE, Gentex, General Motors, Guardian Industries, Guide Corporation, Hella, Honda, Ichikoh Industries, Koito Manufacturing, Labsphere division of X-Rite, Lang-Mekra North America, Magna International, Mitsubushi Motors, Nichia America, North American Lighting, OSRAM Sylvania, Philips Lighting, PPG Industries, Reflec U.S.A., Reflexite, Renault, Samlip, Schefenacker International, Sisecam, Solutia Performance Films, Stanley Electric, TG North America, Toyota Technical Center U.S.A., Valeo, Vidrio Plano, Visteon, 3M Personal Safety Products, and 3M Traffic Control Materials. Information about the Affiliation Program is available at: http://www.umich.edu/ industry

\section{REFERENCES}

Green, P., Kubaki, M., Olson, P. L., \& Sivak, M. (1979). Accidents and the nighttime conspicuity of trucks (Technical Report UM-HSRI-79-92). Ann Arbor: University of Michigan Highway Safety Research Institute.

Knipling, R. R., Mironer, M., Hendricks, D. L., Tijerina, L., Everson, J., Allen, J. C., \& Wilson, C. (1993). Assessment of IVHS countermeasures for collision avoidance: rear-end crashes (Technical report DOT HS 807 995). Washington, DC: United States Department of Transportation National Highway Traffic Safety Administration.

Knipling, R. R., Wang, J.-S., \& Yin, H.-M. (1993). Rear-end crashes: problem size assessment and statistical description (Technical Report DOT HS 807 994). Washington, DC: National Highway Traffic Safety Administration.

Morgan, C. (2001). The effectiveness of retroreflective tape on heavy trailers (NHTSA Technical Report DOT HS 809 222). Washington, DC: National Highway Traffic Safety Administration.

Sullivan, J. M., \& Flannagan, M. J. (2002a). The role of ambient light level in fatal crashes: inferences from daylight saving time transitions. Accident Analysis \& Prevention, 34(4), $487-$ 498.

Sullivan, J. M., \& Flannagan, M. J. (2002b). Some characteristics of pedestrian risk in darkness. Paper presented at the 16th Biennial Symposium on Visibility and Simulation, Iowa City, IA.

Treat, J. R., Tumbas, N. S., McDonald, S. T., Shinar, D., Hume, R. D., Mayer, R. E., Stansifer, N. J., \& Castellan, N. J. (1979). Tri-level study of the causes of traffic accidents: final report (Final Report DOT HS-805 085). Washington, DC: United States Department of Transportation.

Wiacek, C. J., \& Najm, W. G. (1999). Driver/vehicle characteristics in rear-end precrash scenarios based on the General Estimates System (GES) (Technical Report 1999-01-0817). Warrendale, PA: SAE. 